\title{
Adaptación y validación de la CBAS para la observación de climas motivacionales
}

\section{Adaptation and validation of the CBAS for observing motivational climates}

\author{
Adaptação e validação do CBAS para assistir climas motivacionais
}

\author{
Diego Rodríguez-Peláez ${ }^{1}$ Pablo Garrido, Cristina Conde ${ }^{2}$ y Bartolomé J. Almagro ${ }^{3}$
}

1 CEIP Castillo de los Zúniga, 2 Universidad de Huelva y 3 Centro de Estudios Universitarios Cardenal Spinola CEU

Resumen: El objetivo principal del estudio fue adaptar y validar el Coaching Behavior Assessment System (CBAS; Smith, Smoll y Hunt, 1977) para evaluar el clima motivacional que transmite el entrenador. La muestra empleada estuvo compuesta por un único monitor de actividad física dirigida, que trabajaba en el Servicio de Deportes de una universidad pública, tenía 27 años y dos años de experiencia. En primer lugar, se realizó un análisis del contenido de la CBAS por expertos, que propusieron la inclusión de nuevos ítems. En segundo lugar, se llevó a cabo el proceso de entrenamiento de cuatro observadores para evaluar con la adaptación creada de la CBAS. A continuación, los cuatro investigadores realizaron seis observaciones sistemáticas al mismo monitor durante diferentes sesiones elegidas al azar y se calculó el índice de concordancia. Los resultados del análisis de la fiabilidad entre los observadores mostraron valores superiores al $90 \%$, llegando a alcanzar en la última observación el 98,9\%. Se discuten los resultados en base a la importancia de tener una herramienta que nos facilite evaluar el clima motivacional que genera un entrenador a través de la observación sistemática. Palabras claves: motivación, metas de logro, monitor, entrenador, universidad.

Abstract: The main objective of the study was to adapt and validate the Coaching Behavior Assessment System (CBAS, Smith, Smoll \& Hunt, 1977) to assess the motivational climate that conveys the coach. The sample used consisted of a single monitor physical activity directed, who worked at the Sports of a public university was 27 and two years of experience. First, a content analysis of CBAS experts, who proposed the inclusion of new items was performed. Secondly, held training process four observers to evaluate the adaptation set CBAS. Then, the four researchers performed six systematic observations during different sessions randomly and concordance index was calculated. The results of the analysis of the reliability between observers showed values above $90 \%$, reaching the last observation 98.9\%. The results are discussed based on the importance of having a tool that facilitates us to assess the motivational climate that generates a coach through systematic observation.

Keywords: motivation, achievement goals, monitor, coach, college.

Resumo: O principal objetivo do estudo foi adaptar e validar o Coaching Behavior Assessment System (CBAS, Smith, Smoll e Hunt, 1977) para avaliar o clima motivacional que transmite o treinador. A amostra utilizada consistiu em uma atividade física único monitor dirigido, que trabalhou no Esporte de uma universidade pública foi de 27 e dois anos de experiência. Em primeiro lugar, foi realizada uma análise de conteúdo de especialistas CBAS, que propôs a inclusão de novos itens. Em segundo lugar, realizou processo de formação de quatro observadores para avaliar a adaptação definir CBAS. Em seguida, os quatro pesquisadores realizaram observaçôes sistemáticas durante seis sessões diferentes de forma aleatória e índice de concordância foi calculado. Os resultados da análise da fiabilidade entre observadores mostraram valores superiores a $90 \%$, alcançando a última observação de $98,9 \%$. Os resultados são discutidos com base na importância de ter uma ferramenta que nos facilita para avaliar o clima motivacional que gera um treinador por meio de observação sistemática.

Palavras-chave: motivação, metas de realização, monitor, treinador, faculdade.

\section{Introducción}

El entrenador ha de ser considerado como una de las piezas clave en todo proceso de enseńanza-aprendizaje. La comprensión de los determinantes de la motivación en el deporte por parte de sus participantes es uno de los temas que más investigación ha generado en psicología del deporte en los últimos años. Algunos autores afirman que estos determinantes están directamente relacionado con la interacción del entrenador y participantes (Cruz, Torregrosa, Sousa, Mora, y Viladrich, 2010). Dentro de las teorías cognitivo-sociales de la motivación que se aplican al deporte, la teoría de las metas de logro (Achievement Goal Theory) está conjuntamente relacionada

\footnotetext{
Dirección para correspondencia [Correspondence address]: Bartolomé J. Almagro Torres, C/ Ballena, nº 35, Bajo E, 21100. Punta Umbría, Huelva (España). Email: balmagro@ceuandalucia.es
}

con la teoría de la (Self-Determination Theory) predominante en buena parte de dicha investigación (Torregrosa et al., 2011).

El clima motivacional que el entrenador o monitor transmite puede influir de forma determinante en la actitud de los participantes, ya que representa un conjunto de señales implícitas, y/o explícitas, percibidas en el entorno, a través de las cuales se concretan las claves de éxito y fracaso (Smith, Smoll, y Cumming, 2007). Puede implicar a la tarea, fomentando el aprendizaje y el esfuerzo personal, o implicar al ego, fomentando la comparación y superación de unos a los otros (Ames, 1992). De forma general, la literatura apoya que el predominio de un clima u otro, determina unas consecuencias sobre la motivación y, asimismo, sobre el compromiso ha- 
cia la práctica (p.e., Almagro, Sáenz-López, González-Cutre, y Moreno-Murcia, 2011; Leo, Sánchez, Sánchez, Amado, y García-Calvo, 2009).

Tal y como nos señalan Torres, García-Mas, Palmer, y Cruz (2008) existen diversas herramientas sistematizadas para el registro y análisis de los comportamientos desarrollados por los entrenadores en sus interacciones con los jugadores. Una de las más utilizadas, en las últimas décadas, ha sido el Coach Behavioral Assessment System (CBAS) de Smith, Smoll y Hunt (1977). El CBAS es un instrumento de evaluación que se desarrolló a partir de la observación de la actuación de entrenadores en diferentes deportes (béisbol, baloncesto y fútbol americano) y que permite la observación directa y codificada de la conducta del entrenador durante los partidos y los entrenamientos. El CBAS ha demostrado ser un instrumento útil para registrar la mayoría de conductas de los entrenadores, con una elevada fiabilidad entre observadores y capaz de detectar diferencias individuales en los patrones conductuales de los entrenadores (Torres et al., 2008). Además, numerosos estudios (p.e., Mora, Cruz, y Torregrosa, 2009; Sousa, Cruz, Torregrosa, Vilches, y Viladrich, 2006; Wallhead, y Ntoumanis, 2004) han demostrado su eficacia.

El Sistema de Evaluación de la Conducta del Entrenador (CBAS, Smith et al., 1977) se creó para registrar los comportamientos de los entrenadores, pero no específicamente para evaluar o diferenciar entre clima motivacional que implica a la tarea o al ego. En este sentido, el objetivo principal fue adaptar el Coaching Behavior Assessment System (CBAS) de Smith et al. (1977) para evaluar qué clima motivacional transmite el entrenador. En un estudio previo, fue empleado por Conde, Almagro, Sáenz-López, Domínguez y MorenoMurcia (2010) para analizar y comparar la influencia de un programa de formación sobre los climas motivacionales transmitidos por entrenadores de baloncesto. A través de la observación del entrenador y de un programa de formación individualizado, se pretendía disminuir aquellas conductas que implicaban al ego y aumentar las que favorecen el compromiso deportivo de los participantes, es decir, aquellas que implican a la tarea.

Con el fin de profundizar en la observación de la transmisión de climas motivacionales por parte del entrenador (hacia la tarea o al ego), se plantea realizar una serie de modificaciones en el instrumento. Para ello, se intentará demostrar su validez intra e inter-observador a través de porcentaje de acuerdos utilizada por Bellack, Kliebard, Hyman y Smith (1966).

\section{Método}

\section{Participantes}

La muestra utilizada estuvo compuesta por un único monitor de 27 años, de actividades dirigidas, concretamente de GAP (actividad física dirigida enfocada principalmente al trabajo de glúteos, abdomen y piernas). Dicho monitor impartía las clases en el Servicio de Deportes de la Universidad de Huelva. Tenía una experiencia de 2 años en este tipo de actividades y una formación académica relacionada con la enseñanza de la actividad física y el deporte, con el título de Maestro Especialista en Educación física.

\section{Instrumentos}

La recogida de datos se ha llevado a cabo mediante la observación sistemática. El comportamiento del profesor fue analizado a través de una adaptación del Coaching Behavior Assessment System (CBAS) de Smith et al. (1977), el cual se ha mostrado como un instrumento útil para la medición (Conde et al., 2010; Mora et al., 2009; Wallhead y Ntoumanis, 2004). Se utilizó una adaptación de la versión de la CBAS aplicadas por Conde et al. (2010), la cual medía 12 categorías de la conducta del entrenador, dividida en dos dimensiones: reactivas y espontáneas. Las reactivas son provocadas por la realización de la actividad de los jugadores basándose en sus aciertos, errores o conductas. Por otro lado, las espontáneas son respuestas iniciadas por el entrenador (véase Tabla 1). Atendiendo a la CBAS se considera como orientación hacia la tarea el registro de: Refuerzo (R), Ánimo al Error (AE), Mantener el Control (MC), Instrucción Técnica General (ITG), Comunicación General (CG), Organización (O) y Ánimo General (AG). Mientras que se considera como enfoque ego el registro de: No Refuerzo (NR), Punición/Castigo (P), Ignorar el Error (IE) e Instrucción Técnica Punitiva (ITP). 
Tabla 1. Adaptaciones realizadas a la CBAS por Conde et al. (2010).

\begin{tabular}{|c|c|}
\hline $\begin{array}{c}\text { CBAS ORIGINAL } \\
\text { (Smith, Smoll y Hunt, 1977) }\end{array}$ & $\begin{array}{l}\text { ADAPTACIÓN CBAS } \\
\text { (Conde et al., 2010) }\end{array}$ \\
\hline Conductas Reactivas & Conductas Reactivas \\
\hline $\begin{array}{l}\text { Respuestas a conductas deseables } \\
\text { - } \text { Refuerzo (R) } \\
\text { - No Refuerzo (NR) }\end{array}$ & $\begin{array}{l}\text { Respuesta a ejecuciones correctas } \\
\text { 1. Refuerzo positivo verbal o no verbal } \\
\text { 2. No refuerzo }\end{array}$ \\
\hline $\begin{array}{l}\text { Respuesta a los errores } \\
\text { - Ánimo al error (AE) } \\
\text { - Instrucción Técnica al Error (ITE) } \\
\text { - Punición/ Castigo (P) } \\
\text { - Instrucción Técnica Punitiva (ITP=ITE+P) } \\
\text { - Ignorar el Error (IE) }\end{array}$ & $\begin{array}{l}\text { Respuesta a ejecuciones incorrectas } \\
\text { 3. Ánimo } \\
\text { 4. Feedback técnico } \\
\text { - } 4.1 \text { Interrogativo } \\
\text { - } 4.2 \text { Ego } \\
\text { - } 4.3 \text { Tarea } \\
\text { 5. Castigo } \\
\text { 6. Feedback técnica de manera hostil } \\
\text { 7. Ignorar el error }\end{array}$ \\
\hline $\begin{array}{l}\text { Respuesta a comportamientos disruptivos } \\
\text { - Mantener el control (MC) }\end{array}$ & $\begin{array}{l}\text { Respuesta a mala conducta } \\
\text { 8. Mantener el control } \\
\text { - } 8.1 \text { Lo ignora } \\
\text { - } 8.2 \text { No lo ve } \\
\text { - } 8.3 \text { Control } \\
\text { - } 8.4 \text { Control hostil } \\
\end{array}$ \\
\hline Conductas Espontáneas & Conductas Espontáneas \\
\hline $\begin{array}{l}\text { Relacionadas con el partido/entrenamiento } \\
\text { - Instrucción Técnica General (ITG) } \\
\text { - Ánimo General (AG) } \\
\text { - Organización (O) }\end{array}$ & $\begin{array}{l}\text { Aspectos relacionados con la tarea } \\
\text { 9. Instrucción técnica de forma general } \\
\text { - } 9.1 \text { Interrogativo } \\
\text { - } 9.2 \text { Ego } \\
\text { - } 9.3 \text { Tarea } \\
\text { 10. Instrucción de ánimo de forma general } \\
\text { 11. Organización } \\
\text { - } 11.1 \text { Formal } \\
\text { - } 11.2 \text { No formal }\end{array}$ \\
\hline $\begin{array}{l}\text { Irrelevantes para el partido/entrenamiento } \\
\quad \text { - Comunicación General (CG) }\end{array}$ & $\begin{array}{l}\text { Aspectos relacionados con la tarea } \\
\text { 12. Comunicación general } \\
\text { - } 12.1 \text { Inicial } \\
\text { - } 12.2 \text { Final } \\
\end{array}$ \\
\hline
\end{tabular}

\section{Procedimiento}

Se solicitó autorización al Servicio de Actividades Deportivas de la universidad, así como su colaboración para poder realizar la investigación. Además, el estudio fue aprobado por el Comité de Bioética de la Universidad de Huelva.

El primer paso de la adaptación fue la validación del contenido por expertos. Se reunió a cuatro expertos en baloncesto y climas motivacionales quienes propusieron la inclusión de nuevos ítems. A la versión utilizada por Conde et al. (2010), se le añadieron cinco ítems. Uno en el factor MC y cuatro dentro CG (ver Tabla 2). Tres de los ítems incluidos (concretamente, "No existe mala conducta", "CG inicial indagativa" y "CG final indagativa") se incluyeron dentro de los ítems considerados como evocadoras hacia la orientación hacia la tarea. Mientras que los dos ítems restantes ("CG inicial prescriptiva" y "CG final prescriptiva") fueron asumidos como orientadores al ego. 
Tabla 2. Nuevos ítems incluidos en la versión de la CBAS de Conde et al. (2010).

\begin{tabular}{lll}
\hline Respuesta a la mala conducta & & \\
\hline Mantener el control (MC) & 8.1. Lo ignora & Ve la mala conducta y no actúa \\
\cline { 2 - 3 } & 8.2. No lo ve & No se da cuenta \\
\cline { 2 - 3 } & 8.3. Control & Mantiene el control adecuadamente \\
\cline { 2 - 3 } & 8.4. Control hostil & Mantiene el control, con amenazas, enfado, gritando... \\
\cline { 2 - 3 } & 8.5. No existe mala conducta & No existe mala conducta \\
\hline Aspectos relacionados con la tarea & & \\
\hline Comunicación general (CG) & 12.1.1. Inicial indagativo \\
\hline & 12.1.2. Inicial prescriptivo \\
\hline & 12.2.1. Final indagativo \\
\hline
\end{tabular}

En el segundo paso, se comenzó con la fase de entrenamiento de los observadores. Esta fase se llevó a cabo por cuatro observadores, analizando varios entrenamientos filmados. Las dos primeras observaciones se realizaron en voz alzada y de forma grupal por los observadores. Tras el entrenamiento descrito, se le dio a cada observador la grabación de una sesión de entrenamiento que se realizaría atendiendo a los criterios previamente expuestos sin contacto con el resto de observadores. Una vez recopilados los registros individuales se contrastaron los datos obtenidos por cada observador para determinar la fiabilidad intra-observador, obtenida mediante el análisis de frecuencia que determina el índice de concordancia.

Para la validez de contenido se usó el juicio de expertos, siguiendo el metódo Delphi (Landeta, 1999; Steurer, 2011). Según Van Der Fels-Klerx, Gossens, Saaticamp y Horst (2002) este método es de los más adecuados, ofrece un alto nivel de interacción entre los expertos, evitando las desventajas de otros métodos.

Autores como Moreno et al. (2002) o Flick (2007) recomiendan que el proceso de entrenamiento lo lleven a cabo varios observadores, para garantizar mayor fiabilidad. Las observaciones se llevaron a cabo por los cuatro investigadores. La primera reunión fue de familiarización con los ítems. En la segunda reunión se comenzó a observar a voz alzada discutiendo y resolviendo cada una de las dudas. Tras la segunda reunión, se fue observando individualmente y poniendo en común los resultados. Al final de cada reunión se calculó el grado de fiabilidad y cuando se superó el $80 \%$ se consideró que cada miembro podía observar por separado (Goetz y LeCompte, 1988). A continuación, los cuatro investigadores realizaron 6 observaciones sistemáticas al mismo monitor durante diferentes sesiones elegidas al azar.

Por último, hay que destacar que para sistematizar el registro y el análisis de la información, se elaboró una planilla en la que se establecía de manera neta el registro obtenido por el entrenador en cada uno de los ítems. Refiriéndonos a neto como el número de tareas dentro de una sesión que presentan registrado un ítem concreto. En la misma planilla se establecía también el porcentaje obtenido en cada ítem, de las actividades en las que se producía o no alguna intervención del entrenador referente a ese aspecto, estableciendo lo que hemos llamado porcentaje neto.

\section{Análisis de datos}

Para el cálculo de porcentaje de clima ego y clima tarea de cada entrenamiento se realizó un sumatorio del porcentaje neto de cada ítem en relación a las actividades en las que la conducta aparecía al menos una vez, posteriormente se sumó el porcentaje obtenido entre todos los ítems, cuyo resultado conformaría el $100 \%$. Una vez obtenido el total se dividió el sumatorio de los ítems enfocados al ego y los enfocados a la tarea, permitiendo comprobar que porcentaje respecto al total obtenía cada uno determinando de este modo la cantidad en forma porcentual de tarea y ego transmitido durante la sesión.

Se realizó un análisis de frecuencia que determina el índice de concordancia, basado en la fórmula de porcentaje de acuerdos utilizada por Bellack et al. (1966) descrita en la Figura 1.

Figura 1. Fórmula para calcular el índice de concordancia (Bellacka et al., 1966).

Índice de concordancia $=\frac{n^{o} \text { de acuerdos }}{\left(n^{\circ} \text { de acuerdos }+n^{\circ} \text { de desacuerdos }\right)} \times 100$




\section{Resultados}

Como resultado del análisis de contenido de la CBAS original (Smith et al., 1977) y de la adaptación para evaluar climas de Conde et al. (2010), se obtuvo una nueva planilla de observación sistemática que se puede consultar en la Tabla 3.

Esta nueva versión para evaluar los climas motivacionales presenta algunas modificaciones o adaptaciones con respecto a la realizada por Conde et al. (2010). La primera adaptación hace referencia al ítem 7, enunciado como categoría Aparición de la mala conducta. Se ha ańadido el Ítem 7.5, No existe mala conducta, considerando que la no existencia de mala conducta denota una orientación hacia la tarea en detrimento de la orientación al ego (si se produjera mala conducta).
La segunda adaptación hace referencia a la categoría Aspectos relacionados con la tarea, en concreto, se ha añadido el Ítem 12, Comunicación individual, así como sus categorías 12.1. Comunicación inicial indagativa; 12.2. Comunicación inicial prescriptiva; 12.3. Comunicación final indagativa y 12.4. Comunicación final prescriptiva. Se induce que al igual que el Ítem 11 y sus categorías hacen referencia a una orientación hacia la tarea, de la misma forma, la aparición de comunicaciones individuales debe también estar orientada hacia la tarea. Por otro lado, se planteó que si estas charlas son de carácter indagativo estarían más orientadas hacia la tarea en detrimento de las charlas prescriptivas, que se considerarían más orientadas hacia el ego. A pesar de ello, la simple aparición de las charlas individuales se consideró que implicaban hacia la tarea.

Tabla 3. Planilla empleada para realizar las observaciones sistemáticas.

\begin{tabular}{|c|c|c|c|c|c|c|c|c|c|}
\hline \multicolumn{3}{|c|}{ CATEGORÍAS/ACTIVIDADES } & 1 & 2 & 3 & 4 & 5 & $\ldots$ & TOTAL \\
\hline \multicolumn{10}{|c|}{ RESPUESTA A EJECUCIONES CORRECTAS } \\
\hline 1 & \multicolumn{9}{|c|}{ Refuerzo positivo verbal o no verbal } \\
\hline 2 & \multicolumn{9}{|l|}{ No refuerzo } \\
\hline \multicolumn{10}{|c|}{ RESPUESTAS A EJECUCIONES INCORRECTAS } \\
\hline 3 & \multicolumn{9}{|l|}{ Ánimo al error } \\
\hline \multirow[t]{2}{*}{4} & \multirow[t]{2}{*}{ Feedback técnico } & \multicolumn{8}{|l|}{ Interrogativo o pregunta alumnado } \\
\hline & & \multicolumn{8}{|l|}{ Prescriptivo } \\
\hline 5 & \multicolumn{9}{|c|}{ Castigo y Feedback técnico de manera hostil } \\
\hline 6 & \multicolumn{9}{|l|}{ Ignorar el error } \\
\hline \multicolumn{10}{|c|}{ RESPUESTA A LA MALA CONDUCTA } \\
\hline \multirow[t]{5}{*}{7} & \multirow{5}{*}{ Mantener el control } & Lo ignora & & & & & & & \\
\hline & & No lo ve & & & & & & & \\
\hline & & Control & & & & & & & \\
\hline & & Control hostil & & & & & & & \\
\hline & & No existe mala conducta & & & & & & & \\
\hline \multicolumn{10}{|c|}{ ASPECTOS RELACIONADOS CON LA TAREA } \\
\hline \multirow[t]{2}{*}{8} & \multirow{2}{*}{$\begin{array}{l}\text { Instrucción técnica de } \\
\text { forma general. }\end{array}$} & Interrogativo o pregunta alumnado & & & & & & & \\
\hline & & Prescriptivo & & & & & & & \\
\hline 9 & \multicolumn{9}{|c|}{ Instrucción de ánimo de forma general } \\
\hline \multirow[t]{2}{*}{10} & \multirow[t]{2}{*}{ Organización } & Formal & & & & & & & \\
\hline & & Informal & & & & & & & \\
\hline \multicolumn{10}{|c|}{ ASPECTOS NO RELACIONADOS CON LA TAREA } \\
\hline \multirow[t]{4}{*}{11} & \multirow[t]{4}{*}{ Comunicación general } & Inicial indagativo & & & & & & & \\
\hline & & Inicial prescriptivo & & & & & & & \\
\hline & & Final indagativo & & & & & & & \\
\hline & & Final prescriptivo & & & & & & & \\
\hline \multirow[t]{4}{*}{12} & \multirow[t]{4}{*}{ Comunicación individual } & Inicial indagativo & & & & & & & \\
\hline & & Inicial prescriptivo & & & & & & & \\
\hline & & Final indagativo & & & & & & & \\
\hline & & Final prescriptivo & & & & & & & \\
\hline
\end{tabular}


Los resultados del análisis de la fiabilidad o de concordancia de acuerdo entre los observadores se muestran en la Figura 2. Los datos mostraron una fiabilidad de más del $90 \%$. Se mostró una evolución en cuanto a las 6 observaciones, ya que el porcentaje de acuerdos fue aumentando conforme se realizaban más observaciones, llegando a alcanzar en la última observación el 98,9 \%. Sólo se muestra un descenso en la grabación 2, pero nunca bajando del ya comentado $90 \%$.

Figura 2. Evolución del porcentaje de acuerdo de los cuatro observadores.

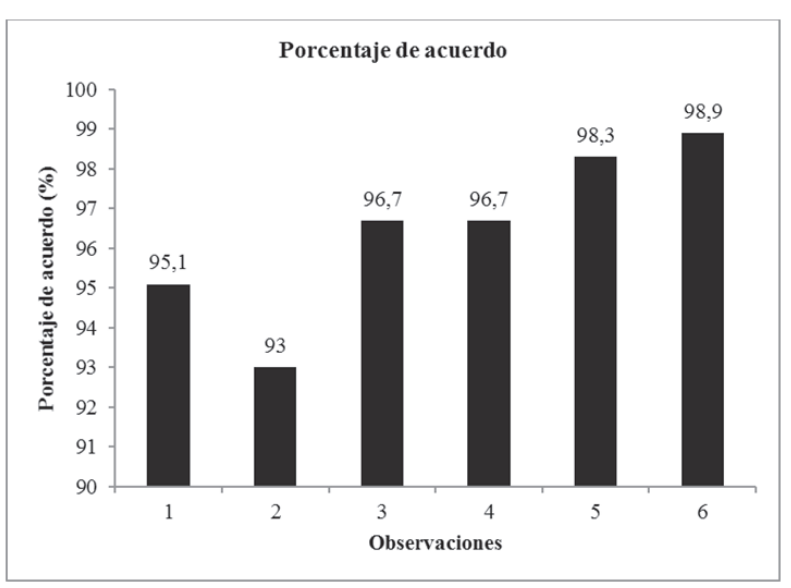

\section{Discusión}

Este estudio trató de adaptar el Coaching Behavior Assessment System (CBAS; Smith et al., 1977) para evaluar el clima motivacional que transmite el entrenador o el monitor de una actividad física dirigida. Para ello, se analizó una primera adaptación de la CBAS realizado en un estudio previo (Conde et al., 2010), que se centró en estudiar la conducta del entrenador en relación con los climas motivacionales que generan y su posible mejora a través de un programa de formación individualizado. En este sentido, los resultados obtenidos mostraron que los cambios introducidos han mejorado la utilidad de la CBAS para analizar el clima motivacional que transmiten los monitores o entrenadores. Además, el porcentaje de acuerdo entre los observadores fue superior al $90 \%$ en las seis sesiones evaluadas.

Los resultados de las observaciones realizadas por los cuatro investigadores en seis sesiones mostraron un porcentaje de acuerdo entre ellos bastante alto. Asimismo, la fiabilidad inter e intra-observadores fue aumentando conforme se realizaban más observaciones, llegando a alcanzar en la última observación el 98,9\%. Sólo se produjo un descenso en la grabación 2, pero nunca bajando del ya comentado $90 \%$. Estos resultados muestran que los cambios introducidos, consensuados por los expertos, no han producido un deterioro sobre la fiabilidad inter e intra-observadores en comparación con los datos que ya aportasen otros estudios que emplearon una adaptación de la CBAS (Conde et al., 2010; Conde, Almagro, Sáenz-López, y Castillo, 2009).

Conocer el clima motivacional que transmite un monitor deportivo es fundamental para poder crear programas de formación personalizados en función de las necesidades de éstos. En esta línea, la investigación (p.e., Conde, 2011; González-Cutre, Sicilia, y Moreno, 2011; Smith et al., 2007) ya ha mostrado los numerosos beneficios que tienen sobre los deportistas/alumnos/practicantes el uso de un clima motivacional que implique a la tarea por parte de los entrenadores/ profesores/monitores. De ahí la importancia de crear o adaptar herramientas que permitan evaluar de manera objetiva el clima motivacional que generan las personas encargadas de dirigir actividades físico-deportivas de cualquier tipo. En este sentido, la adaptación realizada de la CBAS recogida en este manuscrito es un instrumento válido y fiable para ser utilizado en la observación sistemática del comportamiento de los entrenadores, pudiendo distinguir entre conductas que implican hacia la tarea o hacia el ego.

Este trabajo presenta algunas limitaciones que deberán ser subsanadas o tenidas en cuenta en futuros estudios. En primer lugar, sólo se emplearon las observaciones de las sesiones de un mismo monitor deportivo. En segundo lugar, sería interesante testar esta adaptación del CBAS en otros contextos de enseñanza de actividad física o deportiva y con otros observadores distintos. En definitiva, son necesarias más investigaciones que traten de corroborar los resultados obtenidos en otras poblaciones, puesto que la validación es un proceso que no se puede limitar a un solo estudio.

En línea con el objetivo del estudio, se concluye que la hoja de registro CBAS utilizada, a la que previamente se realizó unas modificaciones, resultó un instrumento válido y fiable para analizar el clima motivacional transmitido por un monitor deportivo. La inclusión de los nuevos ítems, además de no interferir en la fiabilidad inter e intra-observadores, puede aumentar la precisión con la que la CBAS evalúa el clima generado por el entrenador. Para que la influencia del entrenador sea positiva sobre la motivación y el compromiso con la práctica deportiva, es fundamental determinar, de forma objetiva, cómo se producen las interacciones en sus clases. Por tanto, el entrenador debe aprender estrategias para fomentar el esfuerzo, la mejora personal, un buen clima de participación y trabajo en grupo, etc.

\section{Aplicaciones prácticas}

De forma concreta, se plantea la importancia del rol del monitor/entrenador como protagonista en el desarrollo de la motivación y de la adherencia a la actividad física de los practicantes. En este sentido, poder analizar el clima motivacional 
que genera el entrenador podrá ayudar a crear programas de formación más adaptados a sus necesidades. En concreto, la adaptación realizada de la CBAS recogida en este manuscrito es un instrumento válido y fiable para ser utilizado en la observación sistemática del comportamiento de los entrenado- res, pudiendo distinguir entre conductas que implican hacia la tarea o hacia el ego. Asimismo, puede contribuir a evaluar si disminuyen aquellas conductas del entrenador que implicaban al ego o si aumentan aquellas que implican a la tarea.

\section{Referencias}

1. Almagro, B. J., Sáenz-López, P., González-Cutre, D., y MorenoMurcia, J. A. (2011). Clima motivacional percibido, necesidades psicológicas y motivación intrínseca como predictores del compromiso deportivo en adolescentes. Revista Internacional de Ciencias del Deporte, 25, 250-265.

2. Ames, C. (1992). Achievement goals, motivational climate and motivational processes. En G. C. Roberts (Ed.), Motivation in sport and exercise (pp. 161-176). Champaign, IL: Human Kinetics.

3. Bellack, A., Kliebard, H., Hyman, R., y Smith, F. (1966). The language of the classroom. Nueva York: Teachers College, Columbia University Press.

4. Conde, C. (2011). Efectos de la intervención en el clima tarea sobre la motivación en jóvenes deportistas. Tesis doctoral. Huelva: Universidad de Huelva.

5. Conde, Almagro, Sáenz-López y Castillo, E. (2009). Intervention and evaluation of the motivational climate transmitted by a basketball coach. Revista de Psicología del Deporte, 18, 357-361.

6. Conde, C., Almagro, B. J., Sáenz-López, P., Domínguez, A., y Moreno, J. A. (2010). Evaluación e influencia de un programa de formación orientado a la mejora del clima motivacional transmitido por entrenadores de baloncesto. Motricidad. European Journal of Human Movement, 25, 165-182.

7. Cruz, J., Torregrosa, M., Sousa, C., Mora, A., y Viladrich, C. (2010). Efectos conductuales de programas personalizados de asesoramiento a entrenadores en estilo de comunicación y clima motivacional. Revista de Psicología del Deporte, 20, 179-195.

8. Flick, U. (2007). Introducción a la investigación cualitativa (2a ed.). Madrid: Morata.

9. Goetz, J. P., y LeCompte, M. D. (1988). Etnografía y diseño cualitativo en investigación educativa. Madrid: Morata.

10. González-Cutre, D., Sicilia, A., y Moreno, J. A. (2011). Un estudio causi-experimental de los efectos del clima motivador tarea en las clases de Educación Física. Revista de Educación, 356, 677-700.

11. Landeta, J. (1999). El método Delphi. Barcelona: Ariel

12. Landis, J. R., y Koch, G. G. (1977). The measurement of observer agreement for categorical data. Biometrics, 33(1), 159-174.

13. Leo, F. M., Sánchez, P. A. Sánchez, Amado, D., y García-Calvo, T.
(2009). Influence of the motivational climate created by coach in the sport commitment in youth basketball players. Revista de Psicología del Deporte, 18, 375-378.

14. Mora, A., Cruz, J., y Torregrosa, M. (2009). Effects of a training program in communication styles of basketball coaches. Revista de Psicología del Deporte, 18, 299-302.

15. Moreno, M. P., Santos, J. A., Ramos, L. A., Sanz, D., Fuentes, J. P., y Del Villar, F. (2002) Aplicación de un sistema de codificación para el análisis de contenido de la conducta verbal del entrenador de voleibol. Motricidad. European Journal of Human Movement, 9, 119-140.

16. Smith, R. E., Smoll, F. L., y Cumming, S. P. (2007). Effects of a motivational climate intervention for coaches on young athletes' sport performance anxiety. Journal of Sport \& Exercise Psychology, 29, 39-59.

17. Smith, R. E., Smoll, F. L., y Hunt, E. B. (1977). A system for behavioral assessment of athletic coaches. Research Quarterly, 48, 401-407.

18. Sousa, C., Cruz, J., Torregrosa, M., Vilches, D., y Viladrich, C. (2006). Evaluación conductual y programa de asesoramiento personalizado (PAPE) a entrenadores de deportistas jóvenes. Revista de Psicología del Deporte, 15(2), 263-278.

19. Steurer, J. (2011). The Delphi Method: an efficient procedure to generate knowledge. Skeletal Radiology 40(8), 959-961.

20. Torregrosa, M., Viladrich, C., Ramis, Y., Azócar, F., Latinjak, A., y Cruz, J. (2011). Efectos en la percepción del clima motivacional generado por los entrenadores y compañeros sobre la diversión y el compromiso. Diferencias en función de género. Revista de Psicología del Deporte, 1 (20), 243-255.

21. Torres, E., García-Mas, A., Palmer, A., y Cruz, J. (2008). El comportamiento observado del árbitro de voleibol y su percepción por parte de las jugadoras: una adaptación preliminar del CBAS. Cuadernos de Psicología del Deporte, 8(2), 5-18.

22. Van Der Fels-Klerx, I. Gossens, L. Saaticamp, H, y Horst, S. (2002). Elicitation of quantitative data from a heterogeneous Expert Panel: Formal process and application in animal health. Risk Analisis, 22(1), 67-81.

23. Wallhead, T. L., y Ntoumanis, N. (2004). Effects of a sport education intervention on students'motivational responses in physical education. Journal of Teaching in Physical Education, 23, 4-18. 